\title{
Effects of incidental training and reinforcement on mixed schema learning ${ }^{1}$
}

A transfer design was used to assess the effects of incidental training and knowledge of results (KR) on mixed schema learning in a reproduction task. The results indicated that schema learning is no faster with $K R$ than without $K R$ and that schemata can be learned when Ss merely attend to the stimuli. The relevance of schema theory to concept formation and other cognitive processes is discussed.

Recent research (Edmonds \& Mueller, 1967a) has demonstrated that in a mixed schema reproduction task Ss readily learn to distinguish among different schemata (recurrent column height sequences) without KR. This finding indicates that in the natural environment humans may spontaneously abstract and use schemata to substantially reduce information processing and storage requirements. Moreover, other data (Edmonds \& Mueller, 1967b) indicate that "what is learned" in perceptual learning are the dimensional or continuous aspects of stimuli (concept of a schema) rather than discrete cues. The relevance of schema theory to such processes as learning to read and transfer of training is thus quite evident (Edmonds \& Mueller, $1967 \mathrm{c})$, but only recently have any systematic parametric investigations been performed to determine the conditions influencing schema learning.

The present study used a transfer design to assess the effects of two relevant variables, incidental training and knowledge of results, on mixed schema learning in a reproduction task.

Subjects

The Ss were 60 undergraduates enrolled in psychology courses at Augusta College. Each $S$ was randomly assigned to one of five equal groups which are referred to as Reinforced (R), Nonreinforced (NR), Nonreinforced Control (NRC), Incidental Learning (I), and Incidental Learning Control (IC).

Patterns

The VARGUS 7 patterns (Evans, 1967) were produced from a seven element Markov process by mapping the elements into column heights. A schema is introduced into each set of patterns by selecting transitional probabilities to favor a most probable sequence (MPS) of column heights; different MPSs constitute different schemata. Each of three different MPSs, designated PS1, PS2, and PS3 was used to produce 67\% redundant patterns containing 12 columns. Selection of these patterns allowed comparisons with data collected in previous experiments. A fourth set (PS4) of 12 column $0 \%$ redundant patterns containing no schema was produced by making all column height sequences equiprobable. The random patterns from PS4 were presented to the two control groups in the training phase of the experiment.

\section{Tasks and Procedure}

In training, Groups $R$ and NR reproduced 15 patterns from each of the three schemata (PS1, PS2, PS3). $A$ randomized block procedure was used in preparing the presentation order of the patterns so that examples of the three schemata were evenly distributed in the sequence. Each pattern was exposed onto a screen for $15 \mathrm{sec}$. After each exposure Ss turned to the appropriate sheet in a mimeographed booklet where the pattern was presented with three columns randomly omitted. The Ss were allowed $30 \mathrm{sec}$ to draw the three omitted columns in each pattern. For Group $R$ each pattern was presented again for 10 sec. Group NR received no KR. The Ss were run in groups of four or less.

The procedure for Groups IC and I were identical to that described for Group NR except Group I used a five point rating scale to judge the area of the 15 patterns from each $67 \%$ redundant PS, while Group IC judged the area of the $450 \%$ redundant patterns (PS4) which contained no schema. In training, Group NRC reproduced the 45 patterns in PS4. The intertrial interval for all groups except Group $R$ was 15 sec; the intertrial interval for Group $R$ was $5 \mathrm{sec}$.

In the testing phase all groups reproduced three patterns from PS1. Patterns from PS2 and PS3 were not also used to assess schema learning since previous data (Edmonds \& Mueller, 1967a) indicated that the three schemata did not differ significantly in difficulty.

Groups IC and NRC, which received training with patterns containing no schema (PS4), were included in the design to eliminate general practice effect as an explanation for improvement in Groups I and NR. For example, the superior performance of Group I as compared to Group IC in the testing phase would indicate that schema learning occurs incidentally (i.e., when Ss merely judge the area of the redundant patterns). Results and Discussion

An analysis of variance based on the number of pattern columns reproduced correctly in the testing phase indicated significant performance differences among the five groups $(F=19.2, d f=4 / 55, p<.001)$. The Tukey (a) procedure (Winer, 1962) was used to test the differences between selected pairs of means. 
The following comparisons were significant at the .01 level: NR $>$ NRC, NR $>$ I, R $>$ I. Group I performed significantly more accurately than Group IC $(p<.025)$. The comparisons between IC and NRC, and NR and $\mathbf{R}$ were nonsignificant.

In brief, these comparisons provide the following information: (1) schema learning occurs in the absence of $\mathrm{KR}(\mathrm{NR}>\mathrm{NRC}$ ) and is no faster with $\mathrm{KR}$ than without KR ( $R=N R)$; (2) schema learning occurs incidentally during irrelevant activity ( $>$ IC); (3) schema learning is faster and more complete under conditions in which it is useful than under conditions in which $S$ judges the area of patterns but cannot benefit from schema learning (NR > I); (4) schema learning is faster and more complete with KR than when $S$ judges the area of patterns in a task that is not aided by schema learning ( $R>I)$.

The nonsignificant performance difference between Groups NR and $R$ has been substantiated by other experiments (Edmonds \& Mueller, 1967c) involving discrimination tasks. These findings indicate that perceptual learning does not conform to an S-R interpretation but consists in an abstraction of the dimensional (schematic) aspects of stimuli. The results of the present study also show that, although incidental learning is not as efficient as intentional learning, humans can learn schemata without any formal instructions.

The question of incidental learning being a misnomer (McLaughlin, 1965) is not involved here. The main point is that schema learning seems to be a basic, automatic process which allows humans, rather independently of variations in motivational level, to abstract and use the common properties of a group of objects.
This abstraction and utilization process allows a considerable reduction in information storage requirements, since only the deviations from the common (schematic) elements must be remembered to reproduce or identify an instance of the schema family.

Another point that should be emphasized concerns the predictability of schema learning. In an experiment involving instances of three schemata mixed together, Edmonds \& Mueller (1967a) found that a best fitting equation describing performance as a function of reproduction trials allowed generalization of results to new Ss and to a new population of patterns differing in schema. Clearly, schema theory has predictive utility under conditions which closely approximate those of the natural environment. Research is currently being performed to determine the influence of other relevant variables on schema learning and schema utilization.

\section{References}

EDMONDS, E. M., \& MUELLER, M. R. Prediction of mixed schema learning in a reproduction task. Psychon. Sci., 1967a, 8, 533-534.

EDMONDS, E. M., \& MUELLER, M. R. The role of schemata in perceptual learning. Psychon. Sci, 1967b, 8, 239-240.

EDMONDS, E. M., \& MUELLER, M. R. Schema discrimination without external reinforcement. Percept. mot. Skills, 1967c, 24, 436-438.

EVANS, S. H. VARGUS 7: Computed patterns from Markov processes. Behav. Sci., 1967, 12, 323-328.

McLAUGHLIN, B. Intentional and incidental learning in human subjects: The role of instructions to learn and motivation. Psychol. Bull, $1965,63,359-376$.

WINER, B. J. Statistical principles in experimental design. New York: McGraw-Hill, 1962.

Note

1. Supported by Office of Education Project No. 6-8338 to the senior author. The authors are grateful to Susan Edmonds for her assistance in the data analysis and to Selby Evans for several suggestions concerning the experimental design. 